\title{
Indolent Retinal Peripheries Attenuate Motion Aftereffect
}

\author{
Ahmad Yousef ${ }^{1}$ \\ ${ }^{1}$ School of Computational Science and Engineering, McMaster University, Hamilton, Ontario, Canada \\ *Correspondence: mohamas2@mcmaster.ca
}

\begin{abstract}
We previously found that the retinal peripheries may not signal the human visual awareness when 'spatial wrapping' stimulus has very low contrast, or when the human subjects perform deep inhalation, see reference 19 and 20. In this study, we found that those techniques are able to significantly reduce the motion aftereffect (MAE) too. Namely, when we reduce the contrast of the MAE stimulator, the MAE is significantly reduced. Similarly, when we ask the subjects to perform deep inhalation in the end of viewing a 'high contrast' MAE stimulator, the MAE is drastically attenuated. The neurophysiological processes of the previous techniques are vastly different, as explained in our previous work. Namely, significant contrast reduction deactivates the retinal peripheries due to the following reasons; first, extremely low contrast stimulus constricts the pupil that disallows the retinal peripheries from receiving enough light rays to signal the brain actively, second, for extremely low contrast conditions, the center-surround antagonism process in the retinal peripheries might not signal the brain at all. Deep inhalation, however, may cause idle links between the retinal peripheries and their corresponding neurological pathways that eventually signal the visual awareness, a process that is also found to weaken the MAE. Introduction

Motion aftereffect is an optical illusion experienced on a stationary stimulus, after fixating on a point in a moving visual stimulus; and it is believed to be the result of motion adaptation. Namely, when one fixate at the center of a rotating spiral for tens of seconds, and then views any stationary pattern, such as brownish yellow grid carpet; the carpet appears to wave in the opposite direction as a result of the spiral aftereffect, see references 1 , and 2 . Important to mention, neural coding mitigate their responses after elongated exposure to a particular type of movement; leading to reduction against the neural resting state potential. Neural adaptation, however, returns the resting state potential to its baseline activities (see references 3,4 , and 5). Interesting, the first human who reported the motion aftereffect (MAE) was Aristotle, but he did not define its direction, see reference 6 . Clear analyses afterwards was provided by Purkyně who observed the MAE after looking at a cavalry parade, see reference 7. Water- fall illusion, which is another form of MAE however, was coined by Thompson, see reference 8 . In this study, we are concerned to see whether the motion aftereffect illusion can be influenced by the two conscious brains theory. In previous studies, we had assumed that each area in the human brain might have a central region, that neurological connected to the central retina, and a peripheral region that is connect to the peripheral retina, see reference 9 , and 10 . Literaturely speaking, retinal peripheries had been believed to be the motion detectors, see reference 11, and 12. Recent studies, however, suggested that motion can be detect by the fovea, but it will be perceived to move slower, see reference, 13. Collectively, the aforementioned facts suggested that the hMT+ region might have two distinct
\end{abstract}

regions, a central region, and a peripheral one. Importantly, when we say hMT+ region might have central and peripheral areas; this doesn't mean these two distinct areas have to be geometrically in the center and the peripheries of hMT+, but they are connected to the fovea and the retinal peripheries respectively. We had been thinking that significant reduction against any visual stimulus may extremely minimize the activation of the retinal peripheries and its corresponding subcortical and cortical brain regions because of the following reasons: extremely low contrast immensely constricts the pupil size, see reference 14; constricted pupils are linked with flat lens and thus smaller 'invert' retinal image, see reference 15; and the signals produced by the center-surround antagonism processes, in extremely low contrast conditions, might be minimal in the retinal peripheries' ganglion cells, see reference 16 .

To study how the inactive retinal peripheries affect the MAE, we significantly reduced the contrast of a spiral stimuli that is assumed to produce massive spiral motion aftereffect. Important to mention, to notice outstanding MAE, human subject has to fixate his/her eye in the central point of the MAE stimulator, see reference 2 . Primarily investigation showed that contrast reduction against the stimulator can significantly attenuate the motion aftereffect illusion. With a specialized timer, the recruited human subjects had noticed that the waving ripples of MAE illusion lasts much lesser 'seconds' with low contrast MAE stimulators.

Materials \& Methods

Twelve human subjects with dilated pupils were recruited to participate in this study. Each human subject has to fixate his/her eyes at a central circle in the MAE stimulator stimuli. Each human subject has to be tested into four sessions; first, the pilot test: that introduces the MAE to the human subject (the MAE stimulator is being sandwiched between two brownish yellow grid carpets to show how the stimulator will ripple the carpet); second, the strength tests: to subjectively measure how the low contrast stimulus affects the MAE amplitude on the brownish yellow grid carpet (the aforementioned test has to be counterbalanced); and finally, the timing tests, in which the human subjects are requested to report the duration of the MAE after high \& low contrast MAE stimulators, see reference 2. Each human subject, using a specialized timer, has to press 1 once the ripples on the brownish yellow grid carpet appear, and then press 2 once the ripples completely disappear. Each stimulus subtends 18.7 DVA in length, and 27.9 in width. The behavioral data were collected manually, and then averaged among the subjects to estimate the simplest statistical values, namely, the average and the standard error of the mean.

Results and Conclusion

As shown in the following diagrams, there is significant degradation against the motion aftereffect normalized strength and its corresponding time duration due to the contrast 


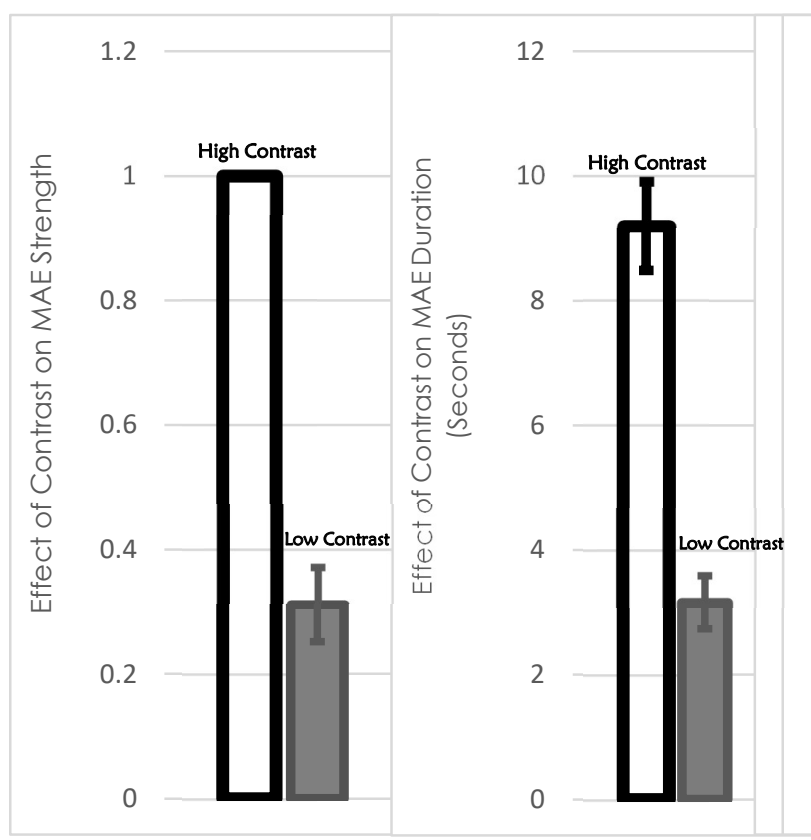

reduction. There is an astonishing correlation between the strength and the time duration of the motion aftereffect; as if the illusion follows the laws of physics, namely, when the strength of fluidic waves is greater, its ripples time duration will last longer. Important to emphasize, these results are consistent with the fact that the neurons which are connected to retinal peripheries return back to their resting state much slower than those neurons which are connected to the fovea, see reference 17. Important to mention, interocular transfer of the MAE had been previously shown an outstanding suppression against the strength MAE, see reference 18 (Box 3). The experiment was outstanding, because it was able to attenuate the motion aftereffect without disabling the retinal peripheries from singling the brain.

Further recent related study; to ultimately defend our claim; we had requested from the twelve human subjects to perform deep inhalation just after the disappearance of the MAE stimulator. As shown in the supplementary materials, unlike the previous setup, the MAE stimulator has highest contrast for every stage; this is because we test whether the retinal peripheries are able to successfully deliver its information to the final neural destinations that signal the consciousness and to structure the global visual awareness. Astonishingly, when the human subjects perform deep inhalation, they noticed much weaker strength of the illusory ripples, and the ripples themselves last much shorter to rest. We were motivated to test the effect of deep inhalation in this study because we think that, at the end of deep inhalation; the oxygenated hemoglobin red blood cells might be reduced in the brain; resulting in reduced activity of hMT+ region which might disallow the emergence of the illusory motion, see reference 20 and 21 . Interesting to mention, these observations has significant meaning, it tells that deep inhalation might be rejecting any illusory spatiotemporal perception regardless the reasons behind the generation.

Essential Notifications

Readers should have direct access to the stimuli, see reference 2. Advisably, download the videos for the best quality. For rapid validation process, have the test with dilated pupil, and watch the videos in moderate environment; namely, fifty percent of the monitor's maximum brightness. Stopwatch could be used to

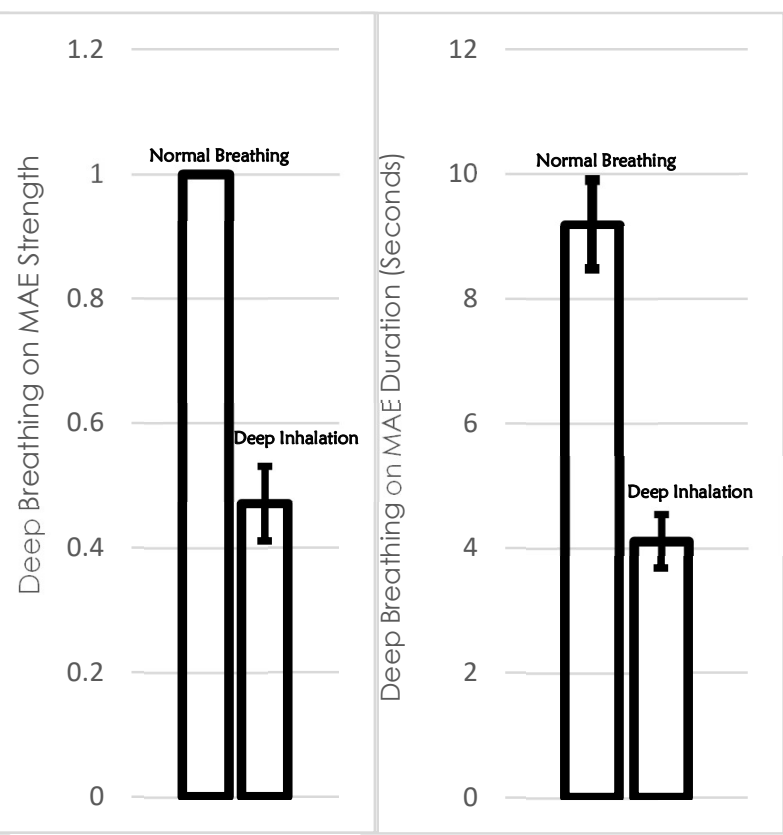

determine the duration of MAE ripples. Scholars with brain imaging facilitations are welcome to collaborate in the aforementioned challenging investigations, namely, to characterize the spatiotemporal properties of the two distinct hMT+ areas, and networking connections the aforementioned brain area with the primary olfactory cortex . Enquiries should be sent to the author.

Transactional References

[1] Broerse, Dodwell, Ehrenstein, (1994). Experiments on the afterimages of stimulus change (Dvorák 1870): a translation with commentary. Perception. [2] You may find the stimuli in the following link:

https://drive.google.com/drive/folders/1UcxECE7sUWLwaKPrsVBaHE3T2dwcUNgF [3] Barlow \& Hill (1963). Evidence for a physiological explanation of the waterfall illusion. Nature.

[4] Srinivasan \& Dvorak (1979). The waterfall illusion in an insect visual system. Vision Research.

[5] Classer, Tsui, Pack \& Tadin (2011). Perceptual and neural consequences of rapid motion adaptation. PNAS Plus.

[6] Aristotle (350 B.C.), Parva Naturalia.

[7] Purkinje. J. (1820). Beiträge zur näheren Kenntniss des Schwindels aus heautognostischen Daten. Medicinische Jahrbücher des kaiserlich-königlichen österreichischen Staates,

[8] Thompson, P. (1880). Optical illusions of motion. Brain.

[9] Yousef, A. (2019). “Two Distinct Fusiform Face Areas.” PsyArXiv. doi:10.31234/osf.io/a8gzv.

[10] Yousef, A. (2019). "Spatial Attention Dilates Time and Length." PsyArXiv. doi:10.31234/osf.io/qazj9.

[11] Finlay, (1982). "Motion perception in the peripheral visual field". Perception.

[12] McKee and Nakayama, (1984). The detection of motion in the peripheral visual field. Vision Research.

[13] Traschütz, Zinke, and Wegener, (2012). Speed change detection in foveal and peripheral vision. Vision Reserach.

[14] Wang , and Munoz. (2014) Modulation of stimulus contrast on the human pupil orienting response, in European Journal of Neuroscience.

[15] Yousef, A (2019). "Linking Dynamics Between Pupil and Lens." engrXiv. doi:10.31224/osf.io/9c3vk.

[16] Rosén; etal. (2014). Quick contrast sensitivity measurements in the periphery. In Journal of Vision.

[17] Purkinje, J. (1825). Neue Beiträge zur Kenntniss des Sehens in Subjectiver Hinsicht. Reimer:Berlin.

[18] Anstis, 1998. The motion aftereffect. Trends in Cognitive Sciences.

[19] Yousef, Ahmad. 2019. "Idle Retinal Peripheries Diminish Spatial Wrapping." PsyArXiv. doi:10.31234/osf.io/srydk.

[20] Yousef, A. 2019. "Deep Inhalation Eliminates Spatial Wrapping." PsyArXiv. doi:10.31234/osf.io/wgjy7.

[21] Yousef, A. 2019. "Deep Breathing Alters Visual Motion Perception." PsyArXiv. doi:10.31234/osf.io/up3sa. 